\title{
Clinical Characteristics and Prognosis of Idiopathic Acute Pancreatitis
}

\author{
Tzvika Porges, M.D. ${ }^{1}$, Tali Shafat, M.D. ${ }^{1,2}$, Iftach Sagy, M.D. ${ }^{1,2,3}$, Dan \\ Schwarzfuchs, M.D. ${ }^{4}$, Ilan Rahmani Tzvi-Ran, M.D. ${ }^{1}$, Alan Jotkowitz, M.D. ${ }^{5}$, and \\ Leonid Barski, M.D. ${ }^{*}$ \\ ${ }^{\prime}$ Internal Medicine Division F, Soroka University Medical Center, Beer-Sheva, Israel; ${ }^{2}$ Clinical Research \\ Center, Soroka University Medical Center, Beer-Sheva, Israel; ${ }^{3}$ Rheumatologic Unit, Soroka University \\ Medical Center, Beer-Sheva, Israel; ${ }^{\star}$ Department of Emergency Medicine, Soroka University Medical \\ Center, Beer-Sheva, Israel; and ${ }^{5}$ Faculty of Health Sciences, Ben-Gurion University of the Negev, Beer- \\ Sheva, Israel
}

\begin{abstract}
Objective: Acute pancreatitis is a serious diagnosis with an increasing incidence in the Western world. In this study we sought to investigate the incidence of idiopathic AP and to compare clinical and prognostic characteristics of idiopathic cases with cases of AP with known etiology.

Methods: In this retrospective study of adult hospitalized patients diagnosed with acute pancreatitis between 2012 and 2015, a comparison was made between admissions of patients with known etiology and those for whom no cause was found. Primary outcome was defined as composite outcome of 30-day mortality and complications.
\end{abstract}

Results: Among 560 admissions of 437 patients with a primary diagnosis of acute pancreatitis, the main factors identified were gallstones (51.2\%) and idiopathic pancreatitis (35.9\%), with alcohol ranked third at only $4.8 \%$. Mortality rate within 30 days of hospitalization was $2.9 \%$ and within one year was $7.1 \%$. Use of

\begin{abstract}
Abbreviations: ACE, angiotensin-converting enzyme; AGA, American Gastroenterological Association; ALT, alanine aminotransferase; AP, acute pancreatitis; ARBs, angiotensin receptor blockers; ERCP, endoscopic retrograde cholangiopancreatography; EUS, endoscopic ultrasound; GEE, generalized estimating equation; HR, hazard ratio; ICU, intensive care unit; IQR, interquartile range; MRCP, magnetic resonance cholangiopancreatography; SD, standard deviation; SUMC, Soroka University Medical Center.
\end{abstract}

Citation: Porges T, Shafat T, Sagy I, Schwarzfuchs D, Rahmani Tzvi-Ran I, Jotkowitz A, Barski L. Clinical Characteristics and Prognosis of Idiopathic Acute Pancreatitis. Rambam Maimonides Med J 2021;12 (3):eoo19.

doi:10.5041/RMMJ.10442

Copyright: (C) 2021 Barski et al. This is an open-access article. All its content, except where otherwise noted, is distributed under the terms of the Creative Commons Attribution License (http://creativecommons.org/licenses/by/3.0), which permits unrestricted use, distribution, and reproduction in any medium, provided the original work is properly cited.

Conflict of interest: No potential conflict of interest relevant to this article was reported.

* To whom correspondence should be addressed. E-mail: lbarski@bgu.ac.il 
lipid-lowering, anti-hypertensive, and anti-diabetic medications was more frequent among patients with "idiopathic" disease (70\%, 68\%, and $33 \%$ versus $59 \%, 56 \%$, and $27 \%$, respectively). Patients admitted with idiopathic AP, in comparison to patients with known AP etiology, had milder disease with shorter hospital stay ( 3 days versus 4 , respectively), and less re-admission in 30 days (7.5\% versus $21.2 \%$ ). Idiopathic AP patients had better prognosis in terms of 30-day death and complication (HR 0.33, 95\% CI 0.08-0.40, $P<0.001$ ).

Conclusion: Idiopathic disease is common among acute pancreatitis patients; the two study groups differed in severity of disease and prognosis. Common use of medications with doubtful value suggests possible under-diagnosis of drug-induced acute idiopathic pancreatitis.

KEY WORDS: Acute pancreatitis, drug induced pancreatitis, idiopathic pancreatitis

\section{INTRODUCTION}

Acute pancreatitis (AP) is an acute inflammatory process of the pancreas. Annual incidence of AP is in the range of $4.9-35$ cases per 100,000 people, with a mortality rate of between $5 \%$ and $10 \%$ of hospitalized patients. ${ }^{1-3}$ In the past few decades there has been an increase of incidence, with a decline in mortality. 4 These trends are probably due to increased prevalence of obesity and alcohol consumption, along with better diagnostic methods and improved medical care. ${ }^{3}$ It is known that the two most common etiologies for AP are obstructive gallbladder disease (up to $40 \%$ of AP cases) and alcohol use (up to $30 \%$ of AP cases).5,6 Additional but less common etiological factors include hypertriglyceridemia, hypercalcemia, and resulting from endoscopic retrograde cholangiopancreatography (ERCP) and drug reaction, among others.

In the absence of etiology it is possible to perform ERCP including aspiration and microscopic examination of the gall contents, magnetic resonance cholangiopancreatography (MRCP), and highresolution endoscopic ultrasound (EUS). These methods can help to diagnose microlithiasis and other possible factors causing AP.7,8 Extensive investigation including advanced imaging techniques such as MRCP, ERCP, EUS, manometry, and gall analysis according to the American Gastroenterological Association (AGA) guidelines still leaves $15 \%-25 \%$ of cases as idiopathic. 5

In a study from Australia published in 2015, the percentage of AP patients with no identified cause was $25.9 \%$; the authors found no significant prognostic difference between these patients and patients with known etiology. 9
In this study we sought to estimate the prevalence of idiopathic AP and to compare clinical and prognostic characteristics of patients hospitalized with an AP diagnosis with known etiology versus idiopathic AP. Furthermore, we intended to follow the group of idiopathic AP patients for a period of one year, to investigate whether etiology for their disease was eventually determined.

\section{METHODS}

\section{Study Population}

This retrospective cohort study included patients aged 18 and above who had been admitted with a primary diagnosis of acute pancreatitis (ICD-9 code 577.0) in Soroka University Medical Center (SUMC), a tertiary hospital that serves one million residents of the southern region of Israel, during the years 2012-2015.

For each admission, demographic and clinical data were collected, including background diseases, chronic medication use, admission diagnosis, hospitalization vitals, and laboratory and imaging results. Two internal medicine physicians reviewed patients' files, and for each case the probable etiology of AP was determined. Cases with no known etiology were defined as idiopathic.

\section{Outcome Measures}

Primary outcome was defined as composite outcome including 30-day mortality, intensive care unit (ICU) admission, complications of pancreatitis (e.g. necrotizing pancreatitis, pseudocyst), surgery due to complications, and 30-day re-admission due to pancreatitis (omitting ambulatory admissions for ERCP or surgery). Secondary outcome was all components of 
the composite outcome, and long-term (1 year) outcome.

\section{Follow-up}

"Idiopathic" pancreatitis patients' medical records were examined for a period of 12 months after discharge in order to ascertain AP etiology in retrospect. During that year, outpatient clinic, imaging, and hospital re-admissions were reviewed.

\section{Statistical Analysis}

Data are expressed as mean \pm standard deviation (SD), median \pm interquartile range (IQR), or number and percentage. We compared patient characteristics between idiopathic versus "non-idiopathic" pancreatitis using $t$ test, chi-square test, and nonparametric test. We used generalized estimating equation (GEE) logistic regression (unstructured matrix) to compare proportions of composite outcome between the two groups. This accounts for the clustering of the same patient composite outcome risk. The final model was selected based on model goodness-of-fit, minimal covariates, and plausible clinical explanation. Statistical analysis was performed using the Statistical Package for Social
Sciences for Windows (SPSS), version 25.0 (IBM Corp, Armonk, NY, USA).

The study was approved by the Institutional Review Board prior to its initiation. Confidentiality was maintained throughout the study.

\section{RESULTS}

\section{Baseline Patient Characteristics}

Between the years 2012 and 2015, there were 560 admissions of 437 patients enrolled in the Israeli health maintenance organization, Clalit Health Services, aged 18 and above to SUMC with a primary diagnosis of AP.

The main probable etiologies were gallstones (51.2\%) and idiopathic AP (35.9\%), with alcohol in third place at only $4.8 \%$ of cases (Figure 1 ). Mean age was 61.9 years, $46.3 \%$ were male, and $86 \%$ were Jewish. The proportion of patients with Charlson comorbidity index $>1$ was $17.7 \%$. Other background diseases are listed in Table 1. According to the modified Glasgow score, $18.4 \%$ of cases were classified as severe, and 3.6\% suffered complications, the most common of which was necrotizing pancreatitis (2.7\%). Of all AP admissions, $2.1 \%$ eventually transferred to the intensive care unit.



Figure 1. Distribution of Probable Acute Pancreatitis Etiology at Hospital Discharge.

ERCP, endoscopic retrograde cholangiopancreatography. 
Table 1. Background Characteristics and Clinical Data of Study Population According to Acute Pancreatitis with Identified versus Unknown Etiology.

\begin{tabular}{|c|c|c|c|c|}
\hline Variable & $\begin{array}{c}\text { Total } \\
(n=560)\end{array}$ & $\begin{array}{l}\text { Identified Etiology } \\
\qquad(n=359)\end{array}$ & $\begin{array}{l}\text { Idiopathic } \\
(n=201)\end{array}$ & $P$ Value \\
\hline \multicolumn{5}{|l|}{ Demographics } \\
\hline Males, $n(\%)$ & $259(46.3)$ & $153(42.6)$ & $106(52.7)$ & 0.022 \\
\hline Age, years, mean $\pm S D$ & $61.9(19.1)$ & $62.5(18.9)$ & $61.0(19.5)$ & 0.376 \\
\hline Jewish, $n(\%)$ & $483(86.3)$ & $307(85.5)$ & $176(87.6)$ & 0.525 \\
\hline Israel born, $n(\%)$ & $217(38.8)$ & $133(37.0)$ & $84(41.8)$ & 0.279 \\
\hline \multicolumn{5}{|l|}{ Background Diseases } \\
\hline Chronic ischemic heart disease, $n(\%)$ & $37(6.6)$ & $26(7.2)$ & $11(5.5)$ & 0.481 \\
\hline Dyslipidemia, $n$ (\%) & $180(32.1)$ & $121(35.1)$ & $59(32.4)$ & 0.563 \\
\hline Chronic obstructive pulmonary disease, $n(\%)$ & $41(7.3)$ & $29(8.1)$ & $12(6.0)$ & 0.402 \\
\hline Diabetes, $n$ (\%) & $159(28.4)$ & $92(25.6)$ & $67(33.3)$ & 0.063 \\
\hline Chronic kidney disease, $n$ (\%) & $72(12.9)$ & $45(12.5)$ & $27(13.4)$ & 0.793 \\
\hline Liver disease, $n(\%)$ & $124(22.1)$ & $88(25.5)$ & $36(19.8)$ & 0.161 \\
\hline Malignancy, $n(\%)$ & $34(6.1)$ & $26(7.5)$ & $8(4.4)$ & 0.194 \\
\hline Charlson co-morbidity index $>1, n(\%)$ & $99(17.7)$ & $61(17.0)$ & $38(18.9)$ & 0.566 \\
\hline Previous admission with pancreatitis, $n(\%)$ & $123(22.0)$ & $83(23.1)$ & $40(19.9)$ & 0.397 \\
\hline \multicolumn{5}{|l|}{ Chronic Medications } \\
\hline ACE/ARBs, $n(\%)$ & $247(44.1)$ & $149(56.0)$ & $98(68.1)$ & 0.020 \\
\hline Diuretics, $n(\%)$ & $88(15.7)$ & $58(21.8)$ & $30(20.8)$ & 0.900 \\
\hline Anti-diabetic drug, $n(\%)$ & $93(16.6)$ & $46(17.3)$ & $47(32.6)$ & 0.001 \\
\hline Lipid-lowering drug, $n$ (\%) & $258(46.1)$ & $157(59.0)$ & $101(70.1)$ & 0.032 \\
\hline \multicolumn{5}{|l|}{ Vital signs on admission } \\
\hline $\mathrm{O}_{2}$ saturation $<90 \%, n(\%)$ & $7(1.3)$ & $1(0.3)$ & $6(3.0)$ & 0.006 \\
\hline \multicolumn{5}{|l|}{ Modified Glasgow Score } \\
\hline Albumin $<3.2 \mathrm{~g} / \mathrm{dL}, n(\%)$ & $83(14.8)$ & $65(18.1)$ & $18(9.0)$ & 0.004 \\
\hline Calcium <8.0 mg/dL, $n(\%)$ & $34(6.1)$ & $27(7.5)$ & $7(3.5)$ & 0.065 \\
\hline Glucose >180 mg/dL, $n(\%)$ & $66(11.8)$ & $41(11.4)$ & $25(12.4)$ & 0.785 \\
\hline $\mathrm{LDH}>600 \mathrm{U} / \mathrm{L}, n(\%)$ & $74(13.2)$ & $54(15.00)$ & $20(10.0)$ & 0.092 \\
\hline Urea >45 mg/dL, $n(\%)$ & $125(22.3)$ & $82(22.8)$ & $43(21.4)$ & 0.751 \\
\hline WBC $>15.0 \times 10^{3} / \mu \mathrm{L}, n(\%)$ & $74(13.2)$ & $51(14.2)$ & $23(11.4)$ & 0.435 \\
\hline $\mathrm{ALT}>150 \mathrm{U} / \mathrm{L}, n(\%)$ & $174(31.1)$ & $135(37.6 \%)$ & $39(19.4)$ & $<0.001$ \\
\hline Modified Glasgow score >2, $n(\%)$ & $103(18.4)$ & $71(19.8)$ & $32(15.9)$ & 0.306 \\
\hline Lipase, $U / L$, mean $\pm S D$ & $737 \pm 1129$ & $850 \pm 1186$ & $615 \pm 1012$ & 0.061 \\
\hline Amylase, $\mathrm{U} / \mathrm{L}$, mean $\pm \mathrm{SD}$ & $451 \pm 534$ & $498 \pm 561$ & $367 \pm 469$ & 0.004 \\
\hline
\end{tabular}

ACE, angiotensin-converting enzyme; ALT, alanine aminotransferase; ARBs, angiotensin receptor blockers; LDH, lactate dehydrogenase; WBC, white blood cell. 


\section{Comparison between "Idiopathic" AP and "Known Etiology" AP}

In the idiopathic patients group, the proportion of male patients was significantly higher $(52.7 \%$ versus $42.6 \%, P=0.022)$, as was the trend of a background diagnosis of diabetes mellitus (33.3\% versus $25.6 \%$ $P=0.063)$. Patients without known etiology were more prone to use medications that interfere with the renin-angiotensin-aldosterone system, as well as anti-diabetic and lipid-lowering medications compared to patients with known etiology.

\section{Outcomes}

Patients with idiopathic disease, as compared to non-idiopathic AP, had milder disease as reflected by shorter hospital length of stay (3 versus 4 days, $P<0.001)$ and decrease of both short (30-day) and long-term (1-year) re-admission rates $(7.5 \%$ versus $21.2 \%, P<0.001$; and $28.3 \%$ versus $46.5 \%, P<0.001$, respectively) (Table 2).

In univariate analysis (Kaplan-Meier curve), idiopathic disease had better outcome according to significant difference in composite outcome (log rank $P<0.001$ ) (Figure 2).

In multivariate analysis, idiopathic disease was associated with better prognosis according to composite outcome (including 30-day mortality, ICU admission, any complication, surgery due to complication, and 30-day re-admission due to pancreatitis) (HR 0.33, 95\% CI $0.08-0.40, P<0.001$, adjusted to Glasgow score, gender, Charlson index, and lipidlowering treatment) (Table 3). Cox regression analysis shows that in addition to having unknown etiology (idiopathic disease), positive endpoints were

Table 2. Prognostic Characteristics According to Acute Pancreatitis with Identified versus Unknown Etiology.

\begin{tabular}{|c|c|c|c|c|}
\hline Characteristic & $\begin{array}{c}\text { Total } \\
(n=560)\end{array}$ & $\begin{array}{l}\text { Identified Etiology } \\
\qquad(n=359)\end{array}$ & $\begin{array}{l}\text { Idiopathic } \\
(n=201)\end{array}$ & $P$ Value \\
\hline \multicolumn{5}{|l|}{ Short-term Outcomes } \\
\hline Admitted to ICU, $n(\%)$ & $12(2.1)$ & $9(2.5)$ & $3(1.5)$ & 0.426 \\
\hline Length of hospital stay, days, median (range) & $4.0(2.0-6.0)$ & $4.0(3.0-6.0)$ & $3.0(2.0-5.0)$ & $<0.001$ \\
\hline Any complication, $n$ (\%), of which: & $20(3.6)$ & $14(3.9)$ & $6(3.0)$ & 0.643 \\
\hline Necrotizing, $n(\%)$ & $15(2.7)$ & $10(2.8)$ & $5(2.5)$ & 0.740 \\
\hline Pseudocyst, $n(\%)$ & $5(0.9)$ & $4(1.1)$ & $1(0.5)$ & \\
\hline Surgery due to complication, $n$ (\%) & $1(0.5)$ & $3(0.8)$ & $0(0.0)$ & 0.194 \\
\hline 30-day re-admission due to pancreatitis, $n$ (\%) & $54(9.6)$ & $49(13.6)$ & $5(2.5)$ & $<0.001$ \\
\hline 30-day re-admission due to any cause, $n(\%)$ & $91(16.3)$ & $76(21.2)$ & $15(7.5)$ & $<0.001$ \\
\hline 30-day ERCP/MRCP tests, $n(\%)$ & $29(5.2)$ & $26(7.2)$ & $3(1.5)$ & 0.002 \\
\hline 30-day mortality, $n$ (\%) & $16(2.9)$ & $11(3.1)$ & $5(2.5)$ & 0.769 \\
\hline Composite outcome, $n(\%)^{*}$ & $86(15.4)$ & $70(19.5)$ & $16(8.0)$ & $<0.001$ \\
\hline \multicolumn{5}{|l|}{ Long-term Outcomes } \\
\hline 365-day mortality, $n(\%)$ & $40(7.1)$ & $25(7.0)$ & $15(7.5)$ & 0.865 \\
\hline 365-day re-admission due to pancreatitis, $n(\%)$ & $112(20.0)$ & $92(25.6)$ & $20(10.0)$ & $<0.001$ \\
\hline 365-day re-admission due to any cause, $n(\%)$ & $224(40.0)$ & $167(46.5)$ & $57(28.4)$ & $<0.001$ \\
\hline 365-day ERCP/MRCP tests, $n(\%)$ & $78(13.9)$ & $52(14.5)$ & $26(12.9)$ & 0.703 \\
\hline Gastro outpatient clinic follow-up, n (\%) & $147(26.3)$ & $80(22.3)$ & $67(33.3)$ & 0.005 \\
\hline
\end{tabular}

* Composite outcome composed of 30-day mortality, ICU admission, any complication, surgery due to complication, and 30-days re-admission due to pancreatitis.

ERCP, endoscopic retrograde cholangiopancreatography; ICU, intensive care unit; MRCP, magnetic resonance cholangiopancreatography. 


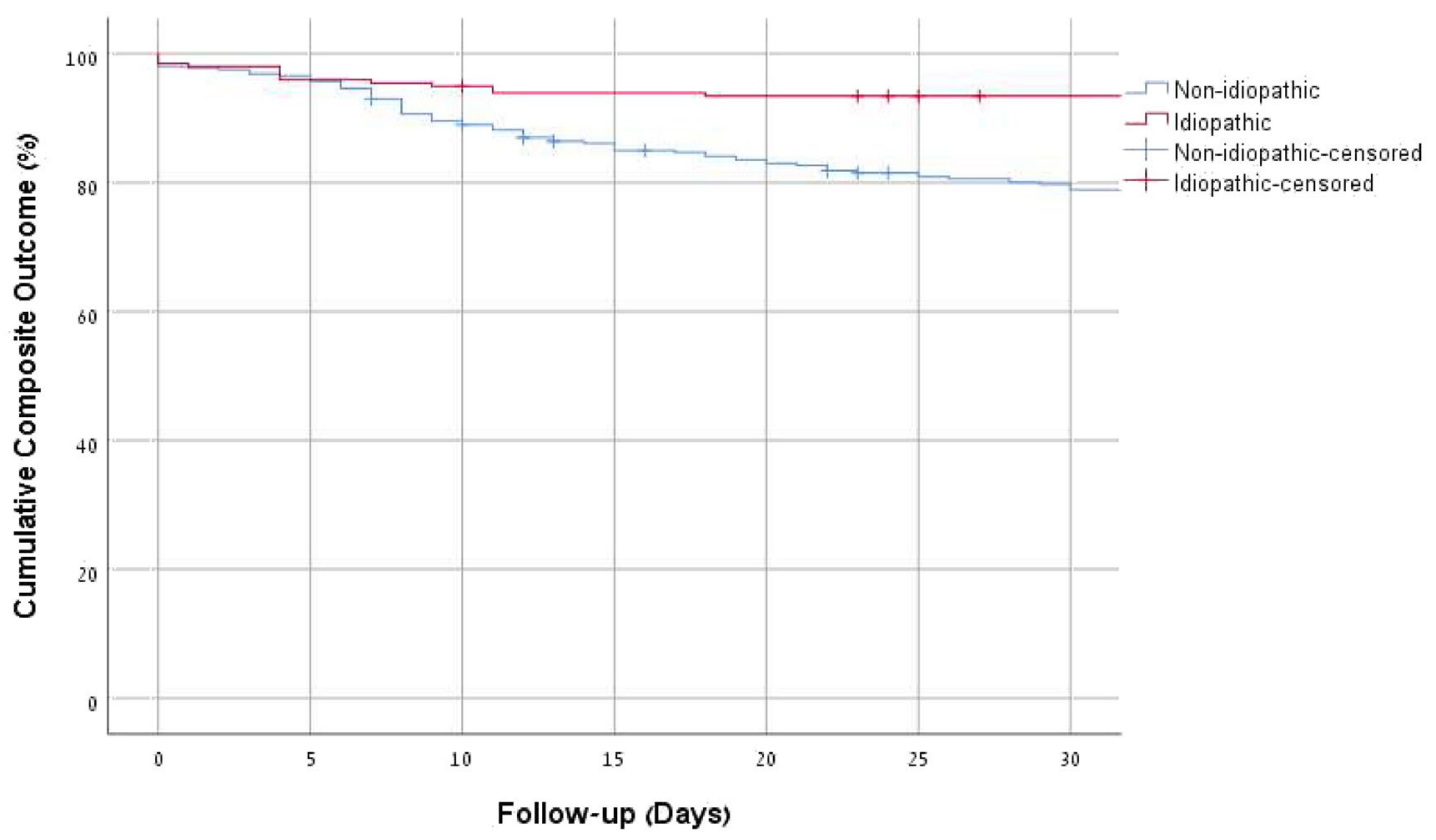

Figure 2. Kaplan-Meier Survival Curve of Composite Outcome (30-day Mortality, ICU Admission, Any Complication, Surgery Due to Complication, and 30-day Re-admission Due to Pancreatitis).

Log rank $P=0.001$.

also significantly more common among patients who used lipid-lowering drugs and had a lower modified Glasgow score (Table 3).

\section{1-Year Follow-up}

During 1-year follow-up of idiopathic disease patients, a probable etiology was found only in 15 of these patients (7.5\%). Distribution of factors is presented in Table 4 .

\section{DISCUSSION}

In this population-based study of hospitalized AP patients, the proportion with idiopathic disease was above one-third. In comparison to known etiology AP patients, patients with idiopathic disease had milder disease with better prognosis in terms of admission stay, and short-term (30-day) and longterm (1-year) re-admissions. Chronic antihypertensive and anti-glycemic medication use was more

com-

Table 3. Multivariate Analysis (Generalized Estimating Model) of Composite Outcome (30-day Mortality, ICU Admission, Any Complication, Surgery Due to Complication, and 30-day Re-admission Due to Pancreatitis).

\begin{tabular}{|lc|c|c|}
\hline Parameter & $\mathrm{HR}$ & $95 \% \mathrm{Cl}$ & $P$ Value \\
\hline Idiopathic disease & 0.33 & $0.08-0.40$ & $<0.001$ \\
Glasgow modified score & 1.51 & $1.21-1.87$ & $<0.001$ \\
Female patient & 0.99 & $0.57-1.71$ & 0.987 \\
Charlson co-morbidity index & 1.15 & $0.76-1.75$ & 0.503 \\
Uses lipid-lowering drugs & 0.41 & $0.23-0.72$ & 0.002
\end{tabular}

$\mathrm{Cl}$, confidence interval; HR, hazard ratio; ICU, intensive care unit. 
Table 4. Idiopathic AP Patients in Study Given New Etiology Subsequent to 12-month Follow-up.

\begin{tabular}{|lc|}
\hline New Etiology $(n=15)$ & Number $(\%)$ \\
\hline Gallstones & $4(26)$ \\
Drug-induced & $4(26)$ \\
Malignancy & $3(20)$ \\
Chronic pancreatitis & $3(20)$ \\
Hypertriglyceridemia & $1(8)$ \\
\hline
\end{tabular}

mon among idiopathic AP patients. It suggests that there is a possibility of underestimation of druginduced pancreatitis.

In our study the most common known cause of AP was gallstones, whereas alcohol consumption was a less prevalent factor. This low prevalence is different compared to most other countries. Previous studies show a variety of factors causing AP, and the variability is explained by differences in lifestyle and habits. Studies have shown that in most European countries and in the United States there are similar rates of AP caused mainly by alcohol and gallstones.5,6,10,11 However, studies carried out in southern European countries such as Greece and Italy show a significant dominance of gallstones as the AP factor.4,12 In this aspect there is a similarity between the population of southern Israel included in this study and the populations of southern Europe/the Mediterranean basin.

The high percentage of patient admissions for idiopathic AP at SUMC during the period of our study may be a result of undiagnosed gallstones, which is recognized as a possible factor in such cases. ${ }^{13,14}$ Additional possibilities are a reporting bias for alcohol consumption and under-diagnosis of drug reactions. ${ }^{15}$

Yet, the percentage of patient admissions for idiopathic AP in our study is similar to findings presented in two large studies carried out within the last 10 years in the United States. ${ }^{16,17}$ In another study $36.5 \%$ of patients whose AP had no identified factor had a higher risk of death. ${ }^{18}$ That study also showed statistical similarities between groups of patients with idiopathic disease and those with biliary disease as factor (predominantly female and older patients). In another study, patients with alcoholic pancreatitis had the highest risk of dying. ${ }^{19}$
This is the first study to suggest a better prognosis in the subgroup of idiopathic AP, which is mainly expressed in shorter length of hospital stay and lower rates of re-admissions. There was also higher modified Glasgow score and more complications among patients with known etiology compared to patients with idiopathic disease, although this difference was not significant.

As mentioned above, patients with idiopathic disease used more angiotensin receptor blockers (ARBs), angiotensin-converting enzyme (ACE) inhibitors, and drugs for diabetes and hyperlipidemia. This significant difference raises the question as to the presence of a causal association between drug use and incidence of idiopathic AP, especially as we note that idiopathic cases tend to be milder than other cases, similar to drug-induced AP which has been shown to have a better prognosis. ${ }^{18}$ Previous studies have shown that drug-induced AP is a less common form of the disease and is thought to make up only $1 \%-2 \%$ of cases. ${ }^{19,20}$

However, the acknowledged difficulty to pinpoint a source in every AP case may mean that druginduced cases are underdiagnosed. We recommend further research to study the association between usage of the aforementioned medications and incidence of AP, in order to support or refute this hypothesis.

The statistical analysis of the study data was carried out not only to determine clinical and prognostic characteristics of idiopathic AP, but also to try and reveal possible etiologies for the disease. Of the patients with idiopathic AP in our study, 33.3\% were followed by a gastroenterology physician, and $12.9 \%$ underwent advanced imaging tests during the year following hospitalization, which led to a small proportion $(7.5 \%)$ of the total number of patients having their disease subsequently reclassified with known etiology.

Previous researchers have proposed that idiopathic cases are actually caused by undiagnosed gallstones and have mentioned various incidence statistics. ${ }^{14,21}$ In order to diagnose whether the source of the illness in these cases is indeed gallstones, various methods can be used: testing for increase in liver enzymes, repeating imaging tests, using advanced imaging such as ERCP, MRCP, and EUS, performing ex vivo bile crystallization, and cholecystectomy. ${ }^{7,8}$ Another common factor in AP is sphincter of Oddi dysfunction, where the assumption is 
that increased pressure on the sphincter may cause AP. ${ }^{22}$ The preferred method of diagnosis in these cases is ERCP plus manometry. ${ }^{23}$ Additional etiological factors revealed by use of invasive procedures include neoplasia and pancreatic divisum. ${ }^{24}$

In our study, several idiopathic cases could be reclassified as biliary according to high liver enzymes (ALT $>150 \mathrm{U} / \mathrm{L}$ ). 25 Only four additional cases (2\%) could be reclassified as caused by gallstones further to data collected from patients during the year following hospitalization. The small number of patients with idiopathic AP who could be reclassified as having gallstones, along with the differences in clinical and prognostic characteristics between groups, creates doubt as to the assumption that the majority of idiopathic cases are actually due to gallstones.

\section{STUDY LIMITATIONS}

This retrospective study was based on data from a single medical center in Israel. We acknowledge possible underestimation of drug use and alcohol consumption as etiological factors, due to reporting bias. Discharged patients were followed for a relatively short period of 12 months.

\section{CONCLUSION}

In conclusion, idiopathic acute pancreatitis is relatively common among AP patients. According to our study, idiopathic AP seems to be less severe than AP with known etiology. We suggest that a partial explanation for this finding could be the underdiagnosis of drug-induced AP, a milder form of the disease, among patients with no identifying factor. This hypothesis is supported by the increased use of certain drugs in the idiopathic AP group. We recommend that efforts be increased to discover the etiology among idiopathic AP patients. Future research should focus on the use of medication as a possible AP factor.

\section{REFERENCES}

1. Sarles H. Revised classification of pancreatitis-Marseille 1984. Dig Dis Sci 1985;30:573.

2. Vege SS, Yadav D, Chari ST. Pancreatitis. In: Talley NJ, Locke GR III, Saito YA, eds. Blackwell GI Epidemiology. 1st ed. Malden, MA, USA: Blackwell; 2007: 221-5.
3. Toouli J, Brooke-Smith M, Bassi C, et al. Guidelines for the management of acute pancreatitis. J Gastroenterol Hepatol 2002;17(Suppl):S15-39. CrossRef

4. Cavallini G, Frulloni L, Bassi C, et al. Prospective multicentre survey on acute pancreatitis in Italy (ProInf-AISP): results on 1005 patients. Dig Liver Dis 2004;36:205-11. CrossRef

5. Forsmark CE, Baillie J; AGA Institute Clinical Practice and Economics Committee; AGA Institute Governing Board. AGA Institute technical review on acute pancreatitis. Gastroenterology 2007;132:2022-44. CrossRef

6. Yang AL, Vadhavkar S, Singh G, Omary MB. Epidemiology of alcohol-related liver and pancreatic disease in the United States. Arch Intern Med 2008; 168:649-56. CrossRef

7. Buscail L, Escourrou J, Delvaux M, et al. A microscopic examination of bile directly collected during endoscopic cannulation of the papilla. Utility in patients with suspected microlithiasis. Dig Dis Sci 1992;37:116-20. CrossRef

8. Yusoff IF, Raymond G, Sahai AV. A prospective comparison of the yield of EUS in primary vs. recurrent idiopathic acute pancreatitis. Gastrointest Endosc 2004;60:673-8. CrossRef

9. Nesvaderani M, Eslick GD, Vagg D, Faraj S, Cox MR. Epidemiology, aetiology and outcomes of acute pancreatitis: a retrospective cohort study. Int J Surg 2015;23(Pt A):68-74. $\underline{\text { CrossRef }}$

10. O’Farrell A, Allwright S, Toomey D, Bedford D, Conlon K. Hospital admission for acute pancreatitis in the Irish population, 1997 2004: could the increase be due to an increase in alcohol-related pancreatitis?. J Public Health 2007;29:398-404. CrossRef

11. Beckingham IJ, Bornman PC. ABC of diseases of liver, pancreas, and biliary system: acute pancreatitis. BMJ 2001;322:595-8. CrossRef

12. Gullo L, Migliori M, Oláh A, et al. Acute pancreatitis in five European countries: etiology and mortality. Pancreas 2002;24:223-7. CrossRef

13. Lee SP, Nicholls JF, Park HZ. Biliary sludge as a cause of acute pancreatitis. N Engl J Med 1992;326: 589-93. CrossRef

14. Ros E, Navarro S, Bru C, Garcia-Pugés A, Valderrama R. Occult microlithiasis in 'idiopathic' acute pancreatitis: prevention of relapses by cholecystectomy or ursodeoxycholic acid therapy. Gastroenterology 1991;101:1701-9. CrossRef

15. Stockwell T, Donath S, Cooper-Stanbury M, Chikritzhs T, Catalano P, Mateo C. Under-reporting of alcohol consumption in household surveys: a com- 
parison of quantity-frequency, graduated-frequency and recent recall. Addiction 2004;99:1024-33. CrossRef

16. Chen Y, Zak Y, Hernandez-Boussard T, Park W, Visser BC. The epidemiology of idiopathic acute pancreatitis, analysis of the nationwide inpatient sample from 1998 to 2007. Pancreas 2013;42:1-5. CrossRef

17. Frey CF, Zhou H, Harvey DJ, White RH. The incidence and case-fatality rates of acute biliary, alcoholic, and idiopathic pancreatitis in California, 19942001. Pancreas 2006;33:336-44. CrossRef

18. Lankisch PG, Dröge M, Gottesleben F. Drug induced acute pancreatitis: incidence and severity. Gut 1995; 37:565-7. CrossRef

19. Sekimoto M, Takada T, Kawarada Y, et al. Guidelines for the management of acute pancreatitis: epidemiology, etiology, natural history, and outcome predictors in acute pancreatitis. J Hepatobiliary Pancreat Surg 2006;13:10-24. $\underline{\text { CrossRef }}$
20. Barreto SG, Tiong L, Williams R. Drug-induced acute pancreatitis in a cohort of 328 patients. A singlecentre experience from Australia. JOP 2011;12:581-5. CrossRef

21. Venu RP, Geenen JE, Hogan W, Stone J, Johnson GK, Soergel K. Idiopathic recurrent pancreatitis. An approach to diagnosis and treatment. Dig Dis Sci 1989;34:56-6o. $\underline{\text { CrossRef }}$

22. Fazel A, Geenen JE, MoezArdalan K, Catalano MF. Intrapancreatic ductal pressure in sphincter of Oddi dysfunction. Pancreas 2005;30:359-62. $\underline{\text { CrossRef }}$

23. Elta GH. Sphincter of Oddi dysfunction and bile duct microlithiasis in acute idiopathic pancreatitis. World J Gastroenterol 2008;14:1023-6. CrossRef

24. Lehman GA. Acute recurrent pancreatitis. Can J Gastroenterol 2003;17:381-3. CrossRef

25. Tenner S, Dubner H, Steinberg W. Predicting gallstone pancreatitis with laboratory parameters: a meta-analysis. Am J Gastroenterol 1994;89:1863-6. 